\title{
Interslice Distance Measurement
}

National Cancer Institute

\section{Source}

National Cancer Institute. Interslice Distance Measurement. NCI Thesaurus. Code

C116123.

A measurement of the distance between slices within an image sequence, calculated by subtracting the nominal slice thickness from the couch increment. 